\title{
Indikation, Risiko und Erfolge der Magenunterkühlung
}

Podiumsdiskussion in Kassel-Wilhelmshöhe am 22. Februar 1964

Die optimistischen Berichte von Wangensteen u. Mitarb. (aus dem Jahre 1962) über ihre ersten Erfahrungen mit dem von ihnen angegebenen Verfahren der Magenunterkühlung haben in der ganzen Welt großes Aufsehen erregt. Es wurden zwei, hinsichtlich des Temperaturbereichs unterschiedliche Methoden der Hypothermie des Magens entwickelt:

Gastric cooling bei massiven Blutungen aus dem oberen Magen-Darmtrakt und Gastric freezing («Magenvereisung») bei Ulcus-duodeni-Leiden und Refluxoeso-phagitis. Die neuen Verfahren wurden auch in Deutschland von verschiedenen Kliniken aufgegrifi $1 / 8 n$ und erprobt. Unter dem Vorsitz von Professor Demling, Stuttgart, be-richteten folgende Herren über ihre ersten Erfahrungen mit diesen neuen Behand-lungsmethoden: McFarland (Liverpool), Göggel (Frankfurt/Main), Grözinger (Heidelberg), Heinkel (Erlangen), Kricke (Kiel), Nagel (Mainz) und Ottenjann (Stuttgart). Methodik, Indikation, Risiko und Erfolge der Magenunterkühlung wurden eingehend erörtert. In dem Schlußwort des Vorsitzenden, dem alle Teilnehmer zu-stimmten, wurde das Diskussionsergebnis wie folgt zusammengefaßt:

Während das gastric cooling offensichtlich eine Bereicherung der Therapie bei Blutungen aus dem oberen Gastrointestinaltrakt darstellt, kann das Verfahren des gastric freezing noch nicht als ausgereift bezeichnet werden. Folgende Punkte schei-nen mir wichtig, hervorgehoben zu werden:

Die Methode sollte, wenn überhaupt, nur stationär durchgeführt werden und zwar an solchen Kliniken, die gastroenterologisch versiert sind. Röntgenkontrollen sollten nicht nur direkt nach der Prozedur, sondern auch 2 bis 4 Wochen und 3 Mo-nate danach vorgenommen werden. Mit Komplikationen, wie Blutung und Magenulzera, muß man rechnen. Man sollte daher nur solche Patienten auswählen, die operationsbedürftig sind oder gar nur solche, die zwar operationsbedürftig, aber aus irgendwelchen Gründen nicht operationsfähig sind.

Möglicherweise läßt sich die Komplikationshäufigkeit reduzieren, wenn man die Minustemperaturen verringert. Das Einbringen eines Thermoelementes zwischen Ballon und Magenwand vermag keine Auskunft darüber zu geben, ob nicht an irgendeiner Stelle Erfrierungen und Nekrosen eintreten, da die Temperaturunter-schiede an der Magenwand, bedingt durch verschieden intensiven Kontakt mit dem Ballon und differente Durchblutungsverhältnisse von $\mathrm{cm} 2 \mathrm{zu} \mathrm{cm} 2$ groß sind.

Es erscheint notwendig, wenn man das Verfahren beibehalten will, weiterhin tierexperimentell zu klären, wie man die Komplikationshäufigkeit senken kann. Diese Mühe lohnt sich meines Erachtens, da wir neben Versagernauchhervorragende Resultate gesehen haben.

Spätschäden durch Gastritis als mögliche Präkanzerose sind nach gastric freezing kauin zu erwarten, jedenfalls in weit geringerem Ausmaße als durch Magenre-sektion, die praktisch immer zu einer sogenannten Stumpfgastritis führt.

Indikation, Risiko und Erfolge der Magenunterkühlung 
6. Darüber, ob die Säuresenkung länger als einige Monate anhält und ob die Rezi-divneigung beim Ulcus-duodeni-Leiden tatsächlich gemindert wird, ist eine Aussage noch nicht möglich. Insgesamt bietet sich das Paradoxon, daß die Magenvereisung heute noch ein heißes Eisen ist, mit dem umzugehen es der äußersten Behutsamkeit bedarf.

R. Ottenjann, Stuttgart

\section{EDITORIAL}

La pyxigraphie intestinale

L'exploration du contenu de Гintestin grêle a toujours pose des problèmes délicats à Гingéniosité des chercheurs. Depuis une dizaine d'années, de grands progrès ont été realises - sans même compter la biopsie pariétale par aspiration - grace au «tubage protege» de Cattan et Maínguet, où $\Gamma$ on réussit à introduire dans le jejunum Tolive d'une sonde en la protégeant contre toute souillure externe; à la «cinéradiométrie» de Debray et Besançon, qui enregistre simulta-nément diverses mesures physiques; et encore l'«endomotorsonde» de Vantrappen et coll. où la capsule est propulsée le long d'un fil con-ducteur par un micromoteur électrique.

Mais toutes ces méthodes comportent des «collecteurs» relies à Pextérieur, et les véhícules líbres ont fait faire un pas de plus dans ce domaine. La «radiotelemetering capsule» de Farrar (New York, 1957) et l' ' Intestinal-Sender» de Sprung (Dresden, 1958) permettent d'enregistrer les pressions tout au long du tractus intestinal. L' «endo-radiosonde» de Nöller (Heidelberg, 1960) a été surtout utilisée pour mesurer le pH des estomacs d'enfants. Connell et coll. (London, 1960) ont prepare trois types de «radio-pills» qui transmettent par transistor des indications sur la pression, la temperature ou le $\mathrm{pH}$, mais n'ont pu utiliser à ce jour (1963) que les données concernant la pression.

Mais déjà auparavant, J.-P. Perrenoud de Neuchâtel (Suisse) avait realise une capsule capable d`effectuer au moment voulu un prélèvement télêcommandé du contenu intestinal. Les premiers résultats furent publiés ici même (Gastroenterologia, vol. 87, p. 349, 1957), et la méthode baptisée pyxigraphie $(\pi v \xi \hat{i} \varsigma=$ capsule) par son auteur. Depuis, plusieurs perfectionnements techniques ont été apportés, et la 